\title{
Recognition of Diabetic Retinopathy Levels Using Machine Learning
}

\author{
Kanak Kalyani ${ }^{1}$, Rina Damdoo ${ }^{2}$ and Jignyasa Sanghavi ${ }^{3}$ \\ ${ }_{1,2,3}$ Assistant Professor, Shri Ramdeobaba College of \\ Engineering \& Management, Nagpur, India
}

\section{ABSTRACT}

Diabetes is one of the major causes of Diabetic retinopathy (DR), it causes damage to eye retina and may lead to blindness. According to the International Diabetics Federation around 77 million cases of diabetes are registered in India in 2020. It is estimated that around 8.5\% population over 18 years and around 45\% population over 60 years is being affected by diabetes in India. The percentage of people being affected by the diabetes is increasing exponentially and the consequences hamper the human life. Therefore awareness and early detection are the key factors in the prevention of diabetic retinopathy. In this paper, we have presented a survey on the latest work carried by the various researchers in the domain along with their contributions. In this paper we have highlighted various research datasets available to work in this field. In this paper we present a model to predict the various class labels of the diabetic retinopathy. In the proposed paper we use data augmentation methods along with histogram equalization. Randomized Hough Transform is used to find the edges. Deep CNN model is used to classify the images. Accuracy of the classifier is around 95\% with sensitivity around 90\%.

\section{KEY WORDS: DIABETIC RETINOPATHY, NPDR, PDR, CNN, DEEP CNN, RANDOMIZED HOUGH TRANSFORM}

\section{INTRODUCTION}

Diabetic Retinopathy (DR) is one of the diabetic eye infection, which harms the retina of an eye because of diabetes. It may inevitably prompt visual impairment. Notwithstanding these scary insights, research demonstrates that at around 80 to $90 \%$ of the new cases could be reduce if there were legitimate and careful treatment and checking of the eyes in the early stage. The diabetic retinopathy is developed in the person who is suffering from diabetics may a while. Study stated that the person who is suffering from diabetics since four

\section{ARTICLE INFORMATION}

*Corresponding Author: kalyanik@rknec.edu

Received 15th Oct 2020 Accepted after revision 24th Dec 2020

Print ISSN: 0974-6455 Online ISSN: 2321-4007 CODEN: BBRCBA

Thomson Reuters ISI Web of Science Clarivate Analytics USA and Crossref Indexed Journal

\section{Clarivate
Analytics}

NAAS Journal Score 2020 (4.31)

A Society of Science and Nature Publication,

Bhopal India 2020. All rights reserved.

Online Contents Available at: http//www.bbrc.in/

Doi: http://dx.doi.org/10.21786/bbrc/13.14/33 months can have a symptoms of Diabetic retinopathy (DR). That's why early detection and diagnosis plays an important role in the prevention of this hazardous disease. It is considered to be a challenging task because of unawareness about the consequences of diabetic can cause on the other human body parts by the patents of diabetics that may lead into blindness. The blindness caused by the diabetic retinopathy is not a curable disease, which makes permanent blindness.

The rate of diabetic peoples is increasing day by day and the need of automatic detection mechanism for this disease is the today's need. The severity of this disease is classified into two classes as proliferative Diabetic retinopathy (PDR) and non-proliferative Diabetic retinopathy (NPDR). The NPDR is further sub-categorized as Sever NPDR, Moderate NPDR, Mild NPDR and Normal. In order to identify these categories and the severity levels the key feature and a measure is blood vessels, microaneurysms, hard executants and cotton wool spots observed into eyes. In the automatic detection 
and classification mechanism, the eye fundus images are considered and based on these images the classifier has been built in-order to classify the images into its appropriate diabetic retinopathy classes and levels. The Diabetic retinopathy occurs when the sugar level in the eye exceeds the certain level which damages the tiny blood vessels of eye. It equally affects the both of the eyes. The DR has four stages (Mansour, 2017, p. 334; Qummar et al, 2019, p. 150530) as follows:

a. Mild Non-proliferative Retinopathy (MNPDR): Clear swellings in blood vessels are being found in retinal images which is previously tiny and thin.

b. Moderate Non-proliferative Retinopathy (NNPDR): Blockings in the blood vessels which shows clear spike in the fundus images. It may cause due to the damage caused to the blood vessels.

c. Severe Non-proliferative Retinopathy (SNPDR): Complete blockage in the blood vessels that affects the load on some of the unblocked blood vessels. It may leads to retinal loss.

d. Proliferative Retinopathy (PDR): New blood vessels are being created due to complete blockage of all the existing blood vessels that leads to the complete loss of vision.
Related work: The various mechanisms have been developed until now for the early detection and classification of severity leaves of diabetic retinopathy. In this section we have analysed various approaches presented and discussed by the research community in the field of diabetic retinopathy. There are many solutions are proposed using machine learning: NN, Regression, PCA, deep learning: CNN, Image processing based approaches, etc. Qiao, Zhu and Zhou (2020, p. 104292) proposed a system that will analyse the occurrence of microaneurysms in fundus retina images using deep learning with the integration of graphic processing unit that will help to boost a system performance. Classification of the fundus images into a class of diabetic retinopathy or normal retina has been done using semantic segmentation which shows better accuracy than the other existing methods. Kumar and $\operatorname{Kumar}(2018$, p. 359) the author introduce the mechanism for classifier Diabetic retinopathy using linear support vector machine (SVM) along with experimental results with accuracy more than 90\%. In this author also brief about preprocessing and the intermediate process followed to classify the fundus images into an appropriate class. In this for pre-processing morphological and histogram equalization has been used. In the next step of detection of MA, the constraint based adaptive histogram equalization (CBAHE) has been used.

Table 1. Comparison of existing work

\begin{tabular}{|l|c|c|c|}
\hline Author & $\begin{array}{c}\text { Methodology/ } \\
\text { Technique }\end{array}$ & Positives & Negatives \\
\hline Dai et al. (2018, p. 1149) & Deep learning & $\begin{array}{c}\text { high-performance and } \\
\text { low-latency inference and } \\
\text { high accuracy }\end{array}$ & $\begin{array}{c}\text { Need GPU to attend } \\
\text { high accuracy }\end{array}$ \\
\hline $\begin{array}{l}\text { Roychowdhury, Koozekanani } \\
\text { and Parhi (2014, p.1717) }\end{array}$ & $\begin{array}{c}\text { linear support } \\
\text { vector machine }\end{array}$ & $\begin{array}{c}\text { sensitivity and specificity } \\
\text { is more than } 90 \%\end{array}$ & $\begin{array}{c}\text { Not suitable for multi } \\
\text { class diabetic retinopathy }\end{array}$ \\
\hline Cao et al. (2018, p.191) & $\begin{array}{c}\text { Machine Learning (ML) } \\
\text { and PCA based Methods }\end{array}$ & $\begin{array}{c}\text { Able to classify } \\
\text { retinal vasculature }\end{array}$ & $\begin{array}{c}\text { Not able to classify the } \\
\text { retinal data into severity class. }\end{array}$ \\
\hline Walter et al. (2002, p. 1236) & Image processing & $\begin{array}{c}\text { Classifier Accuracy } \\
\text { more than } 90 \%\end{array}$ & Complex data structure \\
\hline
\end{tabular}

Alzami et al(2019, p. 272) author presents fractal analysis as a key component for classification of retinal vasculature. In this fractal analysis fractal dimensions are considered that will help to categorize the retinal data into appropriate class with the help of classifier. In this work the author consider the random forest classifier to categorize the images into diabetic retinopathy or normal retinopathy using the MESSIDOR dataset which is one the scientific dataset that contains multiclass retinal images. Deperloglu and Kose (2018, p. 1) suggested the method of classification of retinal fundus images into appropriate classes based on Image processing and deep learning techniques. For feature selection and extraction image processing is used and based on these techniques appropriate deep learning method is implemented in order to classify the dataset into appropriate classes of retinal fundus images. The CNN based classifier has been used along with HSV, V transform algorithm and histogram equalization as a feature selection method. Finally the Gaussian low pass filter is applied to get the selected features.

Dataset; In this proposed area of work various datasets are made available to the researcher by the various communities around the world. The description of some of the dataset available and used in our research is as follows:

The various stages in the proposed methodology are: Stage 1: In the proposed method the data is augmented by various parameters like rotation, rescaling, thresholding, histogram equalization. 
Stage 2: The edges of the images are detected using Randomized Hough Transform. Randomized Hough
Transform performs exceptionally well when we consider 3-Dimensional Data as per Borrmann et al. (2011)

\begin{tabular}{|c|c|c|}
\hline SNo & Dataset & Link \\
\hline 1 & KAGGLE & https://www.kaggle.com/linchundan/fundusimage1000/data \\
\hline 2 & MESSIDOR & http://www.adcis.net/en/DownloadThirdParty/Messidor.html \\
\hline 3 & FAZ & $\begin{array}{l}\text { http://www.biosigdata.com/\%3fdownload\%3dcolour-fundus- images- } \\
\text { of-healthy-persons-patients-with-diabetic-retinopathy }\end{array}$ \\
\hline 4 & DRIVE & https://drive.grand-challenge.org/ \\
\hline 5 & DIARETDB1 & http://www.it.lut.fi/project/imageret/diaretdb1/ \\
\hline
\end{tabular}

Figure 1: Sample Dataset Images

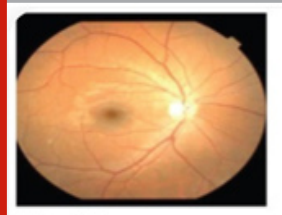

Without DR

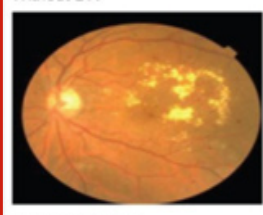

Moderate NPDR

Figure 3: Score of Evaluation parameter

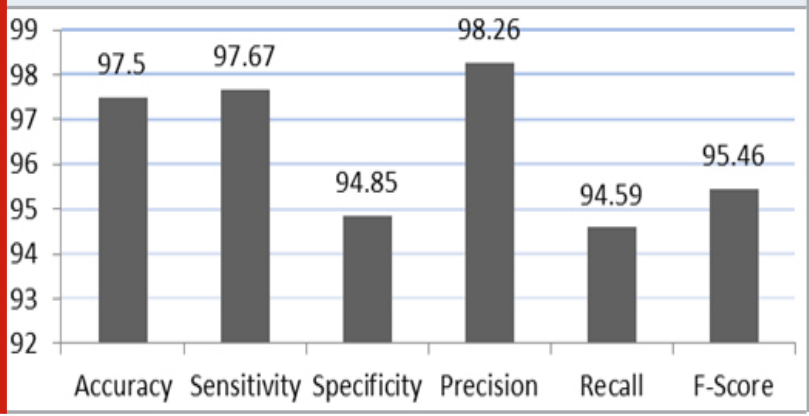

Stage 3: These image are given to Deep convolutional neural network model(DCNN). The DCNN Model is a stack of Convolution, Max-pooling and Dropout layer, which helps to improve the prediction accuracy. The DCNN model consists of following layers:

i. Convolution Layer: Combination of filter and Convolution matrix (Gaussian low pass filter of size $5 \times 5$ to $3 \times 3$ and convolution matrix (256x256 to $4 \times 4)$ )

ii. Max-Pooling Layer: MAX operation over the region $2 \times 2$ of input image.

iii. Supervised Dropout Layer: Performance improvement by preventing the network from over-fitting
Figure 3: Classifier predicted images Normal and Diabetic Retinopathy levels

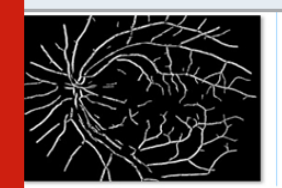

Normal

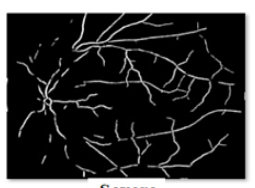

Severe

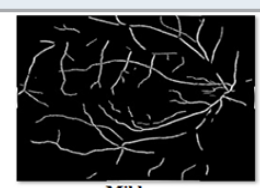

Mild

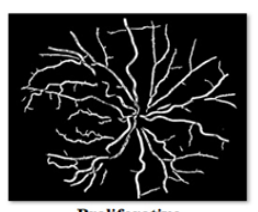

Proliferative

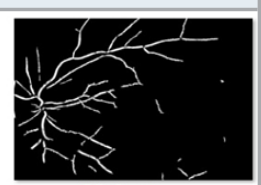

Moderate iv. Hidden Layers and Feature Pooling: Optimization and Rearrangement after Dropout.

v. Activation Function:non-zero gradient rectifier activation function for intermediate layers and softmax probability distribution activation function for output layer.

Output Class Distribution: Proliferative (PDR), Severe, Moderate, Mild Diabetic Retinopathy, Normal/ No Infection.

Prediction Evaluation parameters: Figure 3 shows the classifier output of various predicted classes named as normal, mild, moderate, severe, and proliferative. The images are considered from KAGGLE dataset for classification into appropriate diabetic retinopathy classes. We have also presented the score obtained by our classifier while classification. The proposed classifier is based on the benchmark parameters like Accuracy, Sensitivity, Specificity, Precision, Recall and F-Score. The proposed classifier shows the success rate is around 95\% shown in figure 4.

\section{CONCLUSION}

In this work we provide the various factors and the key points that we need to take care while developing the classifier for the fundus images. We have presented the multilayer machine learning methodology to predict 
the various classes of diabetic retinopathy images along with the detection of blood vessels, spike in the blood vessels caused due to blockage, hard exudates, etc. We have used Randomized Hough Transform for detection of edges which provides better performance when compared to standard Hough transform and generalized Hough transform. The Deep convolutional neural network is used as a key classifier in this proposed work. We have recommended this methodology for deep classifier that predicts hidden classes in the predicted class. Our classifier also able to locate microaneurysms found near to the blood vessels. The proposed methodology has been analysed based on the accuracy, sensitivity, pension, recall and F-score and we found the better results in terms of the analysis of the proposed methodology.

\section{REFERENCES}

Borrmann, D., Elseberg, J., Lingemann, K. et al. "The 3D Hough Transform for plane detection in point clouds: A review and a new accumulator design" 3D Res 2, 3 (2011). https://doi.org/10.1007/3DRes.02(2011)

F. Alzami, Abdussalam, R. A. Megantara, A. Z. Fanani and Purwanto, "Diabetic Retinopathy Grade Classification based on Fractal Analysis and Random Forest," 2019 International Seminar on Application for Technology of Information and Communication (iSemantic), Semarang, Indonesia, 2019, pp. 272-276, doi: 10.1109/ISEMANTIC.2019.8884217.

L. Dai et al., "Clinical Report Guided Retinal Microaneurysm Detection With Multi-Sieving Deep Learning," in IEEE Transactions on Medical Imaging, vol. 37, no. 5, pp. 1149-1161, May 2018, doi: 10.1109/ TMI.2018.2794988.

L. Qiao, Y. Zhu and H. Zhou, "Diabetic Retinopathy Detection Using Prognosis of Microaneurysm and Early Diagnosis System for Non-Proliferative Diabetic Retinopathy Based on Deep Learning Algorithms," in IEEE Access, vol. 8, pp. 104292-104302, 2020, doi: 10.1109/ACCESS.2020.2993937.
Ö. Deperlıglu and U. Köse, ”Diagnosis of Diabetic Retinopathy by Using Image Processing and Convolutional Neural Network” 2018 2nd International Symposium on Multidisciplinary Studies and Innovative Technologies (ISMSIT), Ankara, 2018, pp. 1-5, doi: 10.1109/ISMSIT.2018.8567055.

R. F. Mansour, "Evolutionary Computing Enriched Computer-Aided Diagnosis System for Diabetic Retinopathy: A Survey," IEEE Reviews in Biomedical Engineering, vol. 10, pp. 334-349, 2017, doi: 10.1109/ RBME.2017.2705064.

S. Kumar and B. Kumar, "Diabetic Retinopathy Detection by Extracting Area and Number of Microaneurysm from Colour Fundus Image," 2018 5th International Conference on Signal Processing and Integrated Networks (SPIN), Noida, 2018, pp. 359-364, doi: 10.1109/SPIN.2018.8474264.

S. Qummar et al., "A Deep Learning Ensemble Approach for Diabetic Retinopathy Detection," in IEEE Access, vol. 7, pp. 150530-150539, 2019, doi: 10.1109/ ACCESS.2019.2947484.

S. Roychowdhury, D. D. Koozekanani and K. K. Parhi, "DREAM: Diabetic Retinopathy Analysis Using Machine Learning," in IEEE Journal of Biomedical and Health Informatics, vol. 18, no. 5, pp. 1717-1728, Sept. 2014, doi: 10.1109/JBHI.2013.2294635.

T. Walter, J. -. Klein, P. Massin and A. Erginay, ”A contribution of image processing to the diagnosis of diabetic retinopathy-detection of exudates in color fundus images of the human retina," in IEEE Transactions on Medical Imaging, vol. 21, no. 10, pp. 1236-1243, Oct. 2002, doi: 10.1109/TMI.2002.806290.

W. Cao, N. Czarnek, J. Shan and L. Li, "Microaneurysm Detection Using Principal Component Analysis and Machine Learning Methods," in IEEE Transactions on NanoBioscience, vol. 17, no. 3, pp. 191-198, July 2018, doi: 10.1109/TNB.2018.2840084. 